\title{
Exploring the Local Energy Landscape of Aperiodic Artificial Spin Ices via Lorentz TEM
}

Amanda Petford-Long, Frank Barrows, Vuk Brajuskovic and Charudatta Phatak

Argonne National Laboratory, Lemont, Illinois, United States

As the dimensions of magnetic materials decrease to the nanoscale, novel distributions of spin can be created. We are exploring the behavior of these novel magnetic nanostructures and ways to control the behavior through gaining a quantitative understanding of their local energy landscape as a function of parameters such as structural confinement. Artificial spin ices (ASIs), comprised of magnetic nanoislands or bars patterned on a geometric lattice, are key systems for exploring magnetic frustration [1, 2], a key feature of which is the presence of more than one ground-state configuration. Most work to date has focused on studying periodic artificial spin ice lattices, but there is increasing interest in the behavior of aperiodic quasicrystalline artificial spin ices (QC-ASIs) because they lack translational symmetry and are composed of vertices with a different number of interacting elements, leading to more complicated magnetic frustration. We have explored the behavior of QC-ASIs patterned in $\mathrm{Ni}_{80} \mathrm{Fe}_{20}$ (Permalloy) thin films onto P2 and P3 Penrose quasicrystalline tilings. Patterning was carried out by direct focused ionbeam (FIB) milling of the Permalloy films, which had been deposited directly onto TEM membranes. We use Lorentz transmission electron microscopy (LTEM) and in-situ magnetizing experiments to elucidate the micromagnetic behavior at the nanometer scale. We are able to explore the behavior of these QC-ASIs in different global states (induced by temperature and by demagnetizing protocols) and also to observe their response to applied magnetic fields. Quantitative analysis of the LTEM data is carried out using the transport of intensity equation (TIE) phase retrieval approach to create maps of the in-plane magnetic induction. By combining our experiment with micromagnetic simulations we have explored the local and global energetics of the QC-ASIs. The magnetic configurations used as input for the micromagnetic simulations ate taken directly from our experimental magnetic induction maps. For example, we have explored the way in which aperiodicity leads to local variations in the energy landscape and to a difference in magnetization reversal behavior with respect to periodic ASIs [3]. The QC-ASIs reverse their magnetization via dendritic cascades that form as the magnetization is reversed We are able to calculate the energy at each vertex motif for each possible magnetic configuration from our simulations and thus determine which vertices play the most dominant role in influencing behavior in response to magnetic fields [3, 4]. We were also able to show the emergence of anti-aligned magnetic order during magnetization reversal, and how this depended on the nature of the particular quasicrystal lattice $[5,6]$. 


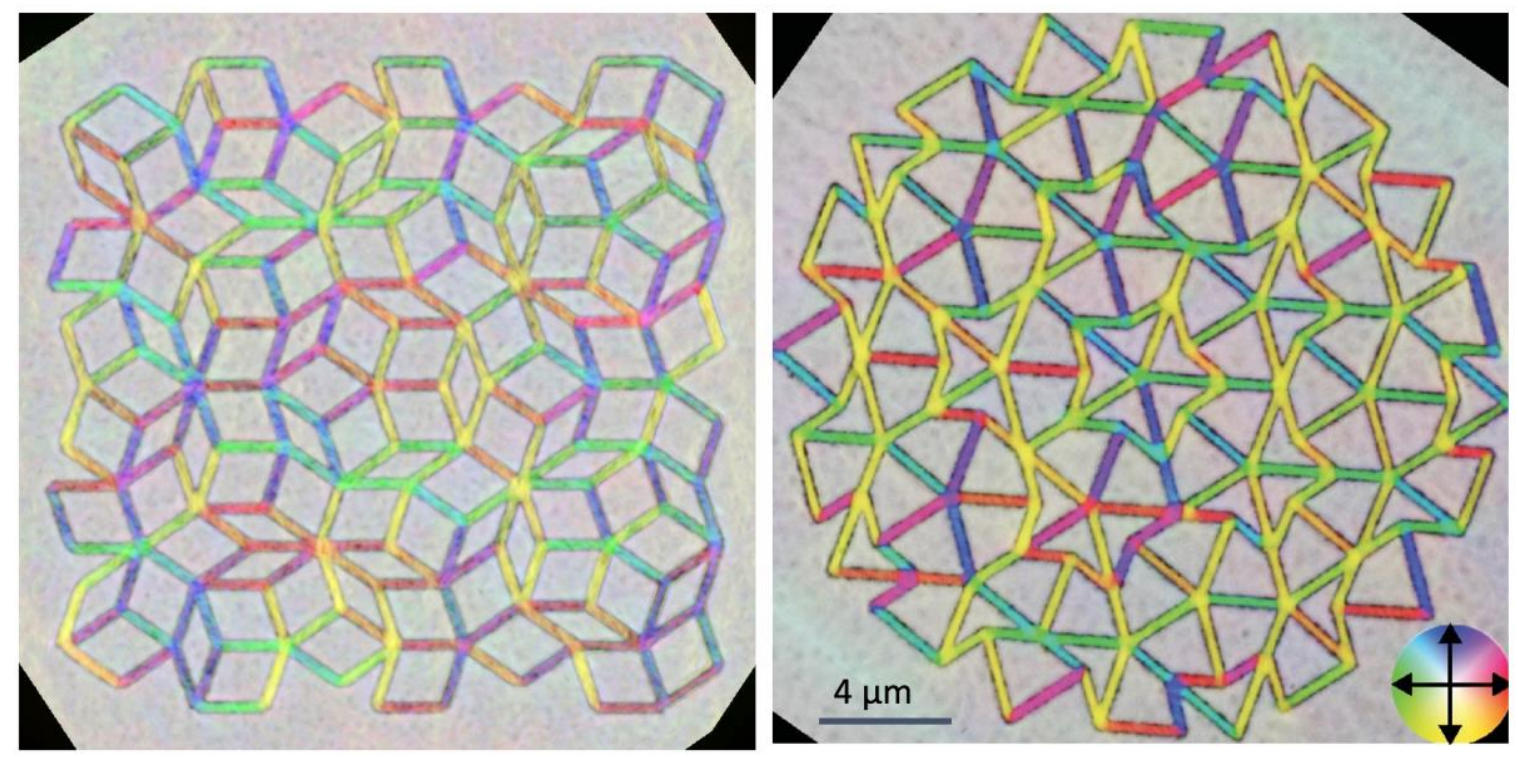

Figure 1. In-plane magnetic induction maps of quasicrystalline artificial spin ices reconstructed from Lorentz TEM data. Left-hand: P3 Penrose tiling and right-hand: P2 Penrose tiling.

\section{References}

1. S. H. Skjærvø, C. H. Marrows, R. L. Stamps, L. J. Heyderman, Advances in artificial spin ice, Nature Reviews Physics 2, 13 (2019).

2. N. Rougemaille and B. Canals, Cooperative magnetic phenomena in artificial spin systems: spin liquids, Coulomb phase and fragmentation of magnetism - a colloquium, European Physics Journal B 92, 62 (2019).

3. V. Brajuskovic, F. Barrows, C. Phatak, A. K. Petford-Long, Real space observation of magnetic excitations and avalanche behavior in artificial quasicrystal lattices, Scientific Reports 6, 34384 (2016).

4. V. Brajuskovic, A. Addi, C. Phatak, A. K. Petford-Long, Observation of transient states during magnetization reversal in a quasicrystal artificial spin ice Phys. Rev. B 98, 094424 (2018).

5. F. Barrows, V. Brajuskovic, A. K. Petford-Long, C. Phatak, Emergent magnetic ordering and topological frustration in quasicrystal artificial spin ices, Physical Review B 99, 094424 (2019).

6. This work was supported by U.S. Department of Energy (DOE), Office of Science, Office of Basic Energy Sciences, Materials Sciences and Engineering Division. Use of the Center for Nanoscale Materials, an Office of Science user facility, was supported by the U.S. Department of Energy, Office of Science, Office of Basic Energy Sciences, under Contract No. DE-AC02-06CH11357. 